\title{
Topology Control Problems Under Symmetric and Asymmetric Power Thresholds
}

\author{
Sven O. Krumke ${ }^{1}$, Rui Liu ${ }^{2}$, Errol L. Lloyd ${ }^{2}$, Madhav V. Marathe ${ }^{3}$, \\ Ram Ramanathan ${ }^{4}$, and S. S. Ravi ${ }^{5}$ \\ 1 Konrad-Zuse-Zentrum für Informationstechnik, Berlin (ZIB), \\ Takustraße 7, 14195 Berlin-Dahlem, Germany. \\ Email: krumke@zib.de. \\ 2 University of Delaware, Newark, DE 19716. \\ Email: $\{$ ruliu, elloyd\}@cis.udel.edu. \\ 3 Los Alamos National Laboratory, MS M997, Los Alamos, NM 87545. \\ Email: marathe@lanl.gov. \\ 4 Internetwork Research Department, BBN Technologies, Cambridge, MA 02138. \\ Email: ramanath@bbn.com \\ 5 University at Albany - SUNY, Albany, NY 12222. \\ Email: ravi@cs.albany.edu.
}

\begin{abstract}
We consider topology control problems where the goal is to assign transmission powers to the nodes of an ad hoc network so as to induce graphs satisfying specific properties. The properties considered are connectivity, bounded diameter and minimum node degree. The optimization objective is to minimize the total power assigned to nodes. As these problems are NP-hard in general, our focus is on developing approximation algorithms with provable performance guarantees. We present results under both symmetric and asymmetric power threshold models.
\end{abstract}

\section{Introduction}

It is well known that battery power is a precious resource in ad hoc networks. Therefore, techniques for minimizing the energy consumed in ad hoc networks have assumed importance. Topology control problems arise in that context. The goal of such problems is to control the topology of networks through the assignment of suitable transmission powers to nodes. Formally, such problems are specified by requiring the induced network to satisfy some graph theoretic properties while minimizing some function of the transmission powers assigned to transceivers (nodes). Previous work in this area has considered properties such as node and edge connectivity and optimization objectives such as minimizing maximum power and minimizing total power. A summary of previous results in this area is presented in Section 3.2.

In this paper, we study topology control problems for three graph properties, namely connectedness, bounded diameter and minimum node degree (Precise formulations of these problems are provided in Section 2.1.). Connectedness is a basic requirement for any network. Ad hoc networks with small diameters are 
desirable in practice since the diameter of a network determines the maximum end-to-end delay for message delivery. Networks in which the degree of each node is at or above a certain threshold value are useful from a reliability perspective. In such networks, the failure of a small number of nodes or links is unlikely to disconnect the network. For all of these properties, the problem of minimizing the maximum power can be solved efficiently; this follows directly from a general result presented in [10]. So, we consider topology control problems for these properties under the objective of minimizing total power. These problems are NP-complete in general. The focus of this paper is therefore on developing approximation algorithms with proven performance guarantees.

Previous work on topology control has assumed the symmetric power threshold model. In that model, the minimum transmission power (also called the power threshold) needed for a node $x$ to reach a node $y$ is assumed to be equal to the minimum transmission power needed for $y$ to reach $x$. In practice, power threshold values for two nodes $x$ and $y$ may be asymmetric because of two reasons. First, the ambient noise levels of the regions containing the two nodes may be different. Secondly, one of the nodes may be equipped with a directional antenna [12] while the other node may have only an omnidirectional antenna. Motivated by these considerations, we study topology control problems under the asymmetric power threshold model. Our results show (as one would expect) that problems do become "harder" under the asymmetric power threshold model. In particular, we show that under the asymmetric power threshold model, the problem of obtaining a connected graph while minimizing the total power cannot be approximated to within a factor of $\Omega(\log n)$, where $n$ is the number of nodes, unless $\mathbf{P}=\mathbf{N P}$. We also present an approximation algorithm with a performance guarantee of $O(\log n)$ for the problem. Under the symmetric power threshold model, it is known that this problem is NP-hard but can be approximated to within a constant factor $[4,8]$.

\section{Problems Considered}

\subsection{Model and Problem Formulation}

We are given a set $V$ of transceivers (nodes). For each ordered pair $(u, v)$ of transceivers, we are given a transmission power threshold, denoted by $p(u, v)$, with the following significance: A signal transmitted by the transceiver $u$ can be received by $v$ only when the transmission power of $u$ is at least $p(u, v)$. It is assumed that $p(u, v)>0$ for all nodes $u$ and $v$.

We study topology control problems under both symmetric and asymmetric power threshold models. Under the symmetric power threshold model, for each pair of transceivers $u$ and $v, p(u, v)=p(v, u)$. The asymmetric threshold model is more general. Under this model, there may be some pairs of transceivers $u$ and $v$ such that $p(u, v) \neq p(v, u)$.

A power assignment is a function $f: V \rightarrow \mathbb{R}^{+}$that specifies a nonnegative power value $f(v)$ to each transceiver $v \in V$. Two models for graphs induced 
by power assignments have been considered in the literature. In this paper we utilize the undirected graph model, in which the induced graph $G_{f}\left(V, E_{f}\right)$ has the undirected edge $\{u, v\}$ if and only if $f(u) \geq p(u, v)$ and $f(v) \geq p(v, u)$. For a power assignment $f$, the maximum power assigned to any node is given by $\max \{f(v): v \in V\}$; the total power assigned to all nodes is given by $\sum_{v \in V} f(v)$.

Following [10], we denote each topology control problem by a triple of the form $\langle\mathbb{M}, \mathbb{P}, \mathbb{O}\rangle$. In such a specification, $\mathbb{M} \in\{$ DIR, UnDIR $\}$ represents the graph model, $\mathbb{P}$ represents the desired graph property and $\mathbb{O}$ represents the minimization objective. In general, $\mathbb{O} \in\{$ MaxP, TotalP $\}$ (abbreviations of Max Power and Total Power respectively). However, for all the problems considered in this paper, $\mathbb{O}=$ TotalP.

Using this notation, we now define the main problems studied in this paper.

1. In the $\langle$ Undir, Diameter, TotalP $\rangle$ problem, we are given a set $V$ of transceivers, the power threshold values $p(u, v)$ for each pair $(u, v)$ of transceivers and a diameter ${ }^{6}$ bound $D$. The goal is to compute a power assignment $f$ such that the undirected graph $G_{f}$ induced by $f$ has diameter at most $D$, and the total power assigned is a minimum among all power assignments that induce graphs satisfying the diameter constraint.

2. In the 〈Undir, Deg LB, TotalP $\rangle$ problem, we are given a set $V$ of transceivers, the power threshold values $p(u, v)$ for each pair $(u, v) \in V$ and an integer $\Delta$, where $2 \leq \Delta \leq|V|-1$. The goal is to compute a power assignment $f$ such that the undirected graph $G_{f}$ induced by $f$ is connected, the degree of each node in $G_{f}$ is at least $\Delta$, and the total power assigned is a minimum among all power assignments that induce connected graphs satisfying the degree constraint.

3. In the 〈Undir, Connected, TotalP $\rangle$ problem, we are given a set $V$ of transceivers and the power threshold values $p(u, v)$ for each pair $(u, v)$ of transceivers. The goal is to compute a power assignment $f$ such that the undirected graph $G_{f}$ induced by $f$ is connected and the total power assigned is a minimum among all power assignments that induce connected graphs.

We study the $\langle$ Undir, Diameter, TotalP $\rangle$ and $\langle$ Undir, Deg LB, TotalP $\rangle$ problems under the symmetric power threshold model. The 〈Undir, ConNECTED, TOTALP $\rangle$ problem has been studied previously under the symmetric power threshold model $[4,8]$. We study it under the asymmetric threshold model (Section 5). Due to space limitations, we discuss only the results for 〈UNDIR, DIameter, TotalP $\rangle$ and 〈Undir, Connected, TotalP $\rangle$ problems in the remainder of this paper.

The following graph theoretic definition is used throughout this paper.

Definition 1. Let $G(V, E)$ be an undirected graph. An edge subgraph $G^{\prime}\left(V, E^{\prime}\right)$ of $G$ uses the same set $V$ of nodes and a subset $E^{\prime}$ of the edge set $E$.

${ }^{6}$ The diameter of $G$, denoted by $\operatorname{DiA}(G)$, is the maximum over the lengths of shortest paths between all pairs of nodes in $G$. 


\subsection{Bicriteria Approximation}

Our results for the diameter problem use the bicriteria approximation framework developed in [11] for dealing with computationally intractable optimization problems involving two objectives. We recall the relevant definitions and notation.

Definition 2. Suppose a problem $\Pi$ with two minimization objectives $A$ and $B$ is posed in the following manner: Given a budget constraint on objective A, find a solution which minimizes the value of objective $B$ among all solutions satisfying the budget constraint. An $(\alpha, \beta)$-approximation algorithm for problem $\Pi$ is a polynomial time algorithm that provides for every instance of $\Pi$ a solution satisfying the following two conditions.

1. The solution violates the budget constraint on objective $A$ by a factor of at most $\alpha$.

2. The value of objective $B$ in the solution is within a factor of at most $\beta$ of the minimum possible value satisfying the budget constraint.

We note that $\langle$ UndiR, Diameter, TotalP $\rangle$ is an example of an optimization problem with two objectives. In this problem, diameter of the induced graph and total power serve as the budgeted objective (with budget $D$ ) and the minimization objective respectively. Thus, an $(\alpha, \beta)$-approximation algorithm for the problem provides a solution where the induced graph has diameter at most $\alpha D$, and the total power assigned is within a factor $\beta$ of the minimum total power needed to induce a graph with diameter at most $D$.

To obtain bicriteria approximation algorithms for the 〈UNDIR, DIAMETER, TOTALP $>$ problem, we rely on known approximation results for another problem, called Minimum Cost Tree with a Diameter Constraint (MCTDC), also involving two minimization objectives. A formal definition of this problem is as follows.

Minimum Cost Tree with a Diameter Constraint (MCTDC)

Instance: A connected undirected graph $G(V, E)$, a nonnegative weight $w(e)$ for each edge $e \in E$, an integer $\delta \leq n-1$.

Requirement: Find an edge subgraph $T\left(V, E_{T}\right)$ of $G$ such that $T\left(V, E_{T}\right)$ is a tree, $\overline{\operatorname{DIA}(T) \leq \delta}$ and the total weight of the edges in $E_{T}$ is the smallest among all the trees satisfying the diameter constraint.

MCTDC is known to be NP-hard [11]. Bicriteria approximations for this problem have been presented in $[2,9,11]$. These results are used in Section 4.

\section{Summary of Results and Related Work}

\subsection{Summary of Results}

The following are the main results presented in this paper. For all the problems, $n$ denotes the number of transceivers in the problem instance. 
1. We show that if the diameter constraint cannot be violated, the 〈UNDIR, DIAMETER, TOTALP $>$ problem cannot be approximated to within an $\Omega(\log n)$ factor unless $\mathbf{P}=\mathbf{N P}$. This result holds even when the diameter bound $D=2$. (Note that the problem is trivial when $D=1$.)

2. We show that using any $(\alpha, \beta)$-approximation algorithm for the MCTDC problem, one can devise a $(2 \alpha, 2(1-1 / n) \beta)$-approximation algorithm for $\langle$ Undir, Diameter, TotalP $\rangle$ problem. This result is based on a general framework presented in [10] for approximating the total power objective. Utilizing this general framework and known bicriteria approximations for the MCTDC problem, we obtain several bicriteria approximation algorithms for the $\langle$ Undir, Diameter, TotalP $\rangle$ problem. (See Section 4.2.)

3. For every fixed integer $\Delta \geq 2$, we show that the $\langle$ Undir, Deg LB, TotalP $\rangle$ problem is NP-complete. Also, we present an approximation algorithm with a performance guarantee of $2(\Delta+1)(1-1 / n)$ for the problem. This algorithm produces a power assignment that induces a connected graph in which each node has degree at least $\Delta$. The performance guarantee is with respect to the optimal total power value. (Details regarding these results will be included in a complete version of this paper.)

4. While the above results are under the symmetric power threshold model, we consider the 〈Undir, Connected, TotalP $\rangle$ problem under the asymmetric power threshold model. We show that the problem cannot be approximated to within an $\Omega(\log n)$ factor unless $\mathbf{P}=\mathbf{N P}$. We also present an $O(\log n)$ approximation algorithm for the problem.

\subsection{Related Work}

Reference [10] provides a general approach that leads to an approximation framework for minimizing total power. Using that framework, two new approximation algorithms for 〈Undir, 2-Node Connected, TotalP $\rangle$ and 〈Undir, 2Edge Connected, TotalP $\rangle$ with an asymptotic approximation ratio of 8 are presented in [10]. Both of the approximation ratios are improved to 4 in [6]. Reference [3] shows that the $\langle$ Dir, Strongly Connected, TotalP $\rangle$ problem is $\mathrm{NP}$-complete and presents a 2-approximation algorithm for the problem. Calinescu et al. [4] improve the approximation ratio to $(1+\ln 2)$. The approximation ratio is further improved to $5 / 3$ in a journal submission based on [4].

\section{Results for Diameter Problems}

\subsection{Lower Bound on Approximation}

The following theorem can be proven using an approximation preserving reduction from the Minimum Set Cover (Msc) problem. The proof is omitted due to space constraint. 
1. From the given problem instance, construct the undirected complete edge weighted graph $G_{c}\left(V, E_{c}\right)$, where the weight of each edge $\{u, v\}$ in $E_{c}$ is equal to the power threshold value $p(u, v)$.

2. Use any approximation algorithm $\mathcal{A}$ for the MCTDC problem on graph $G_{c}\left(V, E_{c}\right)$ with diameter bound $2 D$, and obtain a spanning tree $T\left(V, E_{T}\right)$ of $G_{c}$.

3. For each node (transceiver) $u$, assign a power value $f(u)$ equal to the weight of the largest edge incident on $u$ in $T$.

Fig. 1. Outline of Heuristic Gen-Diameter-Total-Power

Theorem 1. Let $n$ denote the number of nodes in an instance of the 〈UNDIR, DIAMETER, TOTALP $>$ problem. There is a constant $\delta_{1}, 0<\delta_{1}<1$, such that there is no $\delta_{1} \ln n$ approximation for the problem, unless $\boldsymbol{P}=\boldsymbol{N P}$. Moreover, this result holds even for instances in which the diameter bound $D=2$.

\subsection{Bicriteria Approximations for Diameter and Power}

Description of the General Algorithm Recall that the specification for the $\langle$ Undir, Diameter, TotalP $\rangle$ problem includes a bound $D$ on the diameter of the induced graph and that the goal is to minimize total power. Let $n$ denote the total number of transceivers specified in the 〈Undir, Diameter, TotalP $\rangle$ problem instance. Our general approximation algorithm for $\langle$ Undir, DiAmETER, TOTALP $\rangle$, shown in Figure 1, is derived from the general outline presented in [10] for developing approximation algorithms under the total power objective.

In Step 2, we may use any approximation algorithm $\mathcal{A}$ for the MCTDC problem. As long as $\mathcal{A}$ runs in polynomial time, our heuristic also runs in polynomial time. The performance guarantee provided by the heuristic is a function of the performance guarantee provided by Algorithm $\mathcal{A}$.

Performance Guarantee of the General Heuristic The solution produced by Heuristic Gen-Diameter-Total-Power is approximate in terms of both diameter and total power. So, we cannot directly apply the bound from [10] on the performance of the general approach to derive the performance guarantee provided by the heuristic. Our analysis uses a simple property of spanning trees generated by breadth-first-search (BFS).

Throughout Section 4.2, we use the following notation. Let $I$ denote the given instance of the 〈Undir, DiAmeTER, TOTALP $\rangle$ problem with $n$ transceivers and diameter bound $D \geq 1$. Let $f^{*}$ denote an optimal power assignment such that the graph $G_{f^{*}}$ induced by $f^{*}$ has diameter at most $D$, and let $O P T(I)$ $=\sum_{v \in V} f^{*}(v)$. Let $f$ denote the power assignment produced by the heuristic and let $G_{f}$ denote the graph induced by $f$. Let $D T P(I)=\sum_{v \in V} f(v)$, the total power assigned by the heuristic for the instance $I$. The goal of this subsection is to prove the following result. 
Theorem 2. Suppose Algorithm $\mathcal{A}$ used in Step 2 of Heuristic Gen-DiameterTOTAL-Power is an $(\alpha, \beta)$-approximation algorithm for the MCTDC problem. For any instance $I$ of the $\langle\mathrm{UndiR}$, Diameter, TotalP $\rangle$ problem, Heuristic Gen-Diameter-Total-POWER produces a power assignment $f$ satisfying the following two properties.

1. $\operatorname{DiA}\left(G_{f}\right) \leq 2 \alpha D$.

2. $\operatorname{DTP}(I) \leq 2 \beta(1-1 / n) O P T(I)$.

Our proof of Theorem 2 uses a few lemmas proved below. We begin with a simple lemma about spanning trees generated by carrying out BFS on a connected graph. The proof of this lemma is omitted.

Lemma 1. Let $G$ be a connected graph with diameter $\delta$. Let $T$ be any spanning tree for $G$ generated by BFS. Then $\operatorname{Dia}(T) \leq 2 \delta$.

The next lemma indicates why in Step 2 of Heuristic Gen-Diameter-TotalPOWER, we use the diameter bound of $2 D$.

Lemma 2. Consider the complete graph $G_{c}\left(V, E_{c}\right)$ constructed in Step 1 of Heuristic Gen-Diameter-Total-Power. There is a spanning tree $T_{1}\left(V, E_{T_{1}}\right)$ of $G_{c}$ satisfying the following two properties.

(a) $\operatorname{DiA}\left(T_{1}\right) \leq 2 D$.

(b) Let $W\left(E_{T_{1}}\right)=\sum_{\{x, y\} \in E_{T_{1}}} p(x, y)$ denote the total edge weight of $T_{1}$. Then, $W\left(E_{T_{1}}\right) \leq(1-1 / n) O P T(I)$.

Proof:

Part (a): Consider the graph $G_{f^{*}}$ induced by the optimal power assignment $f^{*}$. Note that $\operatorname{DiA}\left(G_{f^{*}}\right) \leq D$. Let $v$ be node such that $f^{*}(v)$ has the largest value among all the nodes in $V$. Let $T_{1}\left(V, E_{T_{1}}\right)$ be a spanning tree of $G_{f^{*}}$ generated by carrying out a BFS on $G_{f^{*}}$ with $v$ as the root. Then, from Lemma 1 , we have $\operatorname{DiA}\left(T_{1}\right) \leq 2 D$.

Part (b): Consider another assignment $w$ of weights to the edges of $T_{1}$ as indicated below. Consider each edge $\{x, y\}$ in $T_{1}$, where $y$ is the parent of $x$. Let $w(x, y)=f^{*}(x)$. Thus, the power value assigned by the optimal solution to each node except the root becomes the weight of exactly one edge of $T_{1}$. The power value $f^{*}(v)$ of the root is not assigned to any edge. Therefore,

$$
\sum_{\{x, y\} \in E_{T_{1}}} w(x, y)=O P T(I)-f^{*}(v) .
$$

Since $v$ has the maximum power value under $f^{*}$ among all the nodes, we have $f^{*}(v) \geq O P T(I) / n$. Therefore,

$$
\sum_{\{x, y\} \in E_{T_{1}}} w(x, y) \leq(1-1 / n) O P T(I) .
$$

The following claim relates the weight $w(x, y)$ to the power threshold value $p(x, y)$. We omit the proof of this claim. 
Claim. For each edge $\{x, y\} \in E_{T_{1}}, w(x, y) \geq p(x, y)$.

As a simple consequence of the above claim, we have

$$
W\left(E_{T_{1}}\right) \leq \sum_{\{x, y\} \in E_{T_{1}}} w(x, y) \leq(1-1 / n) O P T(I),
$$

and this completes the proof of Part (b) of the lemma.

The next lemma, which follows from Lemma 2 , uses the performance guarantee provided by the approximation algorithm $\mathcal{A}$ used in Step 2 of the heuristic.

Lemma 3. Let $T\left(V, E_{T}\right)$ denote the tree produced by $\mathcal{A}$ at the end of Step 2 of Heuristic Gen-Diameter-Total-Power. Let $W\left(E_{T}\right)=\sum_{\{x, y\} \in E_{T}} p(x, y)$ denote the total weight of the edges in $T$. Let $(\alpha, \beta)$ denote the performance guarantee provided by $\mathcal{A}$ for the MCTDC problem. Then,

(a) $\operatorname{DiA}(T) \leq 2 \alpha D$.

(b) $W\left(E_{T}\right) \leq \beta(1-1 / n) O P T(I)$.

We are now ready to prove Theorem 2 .

Proof of Theorem 2: Consider the spanning tree $T\left(V, E_{T}\right)$ produced in Step 2 of the heuristic. We will first show that every edge $\{x, y\} \in E_{T}$ is also in $G_{f}\left(V, E_{f}\right)$, the graph induced by the power assignment constructed in Step 3 of the heuristic. To see this, notice that $f(x)$ is the largest weight of an edge incident on $x$ in $T$. Thus, $f(x) \geq p(x, y)$. Similarly, $f(y) \geq p(x, y)$. Thus, every edge in $E_{T}$ is also in $E_{f}$. Since DiA $(T) \leq 2 \alpha D$, and addition of edges cannot increase the diameter, it follows that $\operatorname{DiA}\left(G_{f}\right) \leq 2 \alpha D$.

To bound $D T P(I)$, we note from Lemma 3 that $W\left(E_{T}\right) \leq \beta(1-1 / n) O P T(I)$. In the power assignment constructed in Step 3, the weight of any edge can be assigned to at most two nodes (namely, the end points of that edge). Thus, the total power assigned to all the nodes is at most $2 W\left(E_{T}\right)$. In other words, $D T P(I) \leq 2 \beta(1-1 / n) O P T(I)$, and this completes the proof of Theorem 2 .

Obtaining Approximation Algorithms from Theorem 2 We now briefly indicate how several bicriteria approximation algorithms for the $\langle$ UNDIR, DIameter, TotalP $\rangle$ problem can be obtained using Gen-Diameter-TotalPOWER in conjunction with known bicriteria approximation results for the MCTDC problem.

1. For any fixed $\epsilon>0$, a $\left(2\left\lceil\log _{2} n\right\rceil,(1+\epsilon)\left\lceil\log _{2} n\right\rceil\right)$-approximation algorithm is presented in [11] for the MCTDC problem. Using this algorithm and setting $\epsilon<1 / n$, we can obtain a $\left(4\left\lceil\log _{2} n\right\rceil, 2\left\lceil\log _{2} n\right\rceil\right)$-approximation algorithm for the $\langle$ Undir, Diameter, TotalP $\rangle$ problem.

2. For any fixed $D \geq 1$, a $(1, O(D \log n))$-approximation algorithm for the MCTDC problem is presented in [2]. Thus, for any fixed $D \geq 1$, we can obtain a $(2, O(D \log n))$-approximation algorithm for the 〈UnDIR, DiAmETER, TOTALP $>$ problem. 
3. For any $D$ and any fixed $\epsilon>0$, a $\left(1, O\left(n^{\epsilon} \log n\right)\right)$-approximation algorithm for the MCTDC problem is presented in [9]. Thus, for this case, we can obtain a $\left(2, O\left(n^{\epsilon} \log n\right)\right)$-approximation algorithm for the 〈Undir, DiameTER, TOTALP $\rangle$ problem.

The above results are for inducing a bounded diameter graph over all the nodes. We can also obtain an approximation algorithm for the Steiner version of the $\langle$ Undir, Diameter, TotalP $\rangle$ problem where only a specified subset of the nodes (called the terminals) need to be connected together into a graph of bounded diameter. Letting $\eta$ denote the number of terminals, reference [11] presents an $(O(\log \eta), O(\log \eta))$-approximation algorithm for the Steiner version of the MCTDC problem. Using this approximation algorithm in Step 2 of Figure 1, we obtain an $(O(\log \eta), O(\log \eta))$-approximation algorithm for the Steiner version of the 〈Undir, Diameter, TotalP $\rangle$ problem.

\section{$5 \quad$ Asymmetric Power Threshold Model Results}

In this section, we consider the $\langle$ UndiR, Connected, TotalP $\rangle$ problem under the asymmetric threshold model. We begin with a lower bound on the approximability of the problem. This lower bound result can be proven in a manner similar to that of Theorem 1.

Theorem 3. Let $n$ denote the number of transceivers in an instance of the $\langle\mathrm{UndiR}, \mathrm{Connected,} \mathrm{TotalP}\rangle$ problem. There is a constant $\delta, 0<\delta<1$, such that there is no $\delta \ln n$ approximation for the problem, unless $\boldsymbol{P}=\boldsymbol{N P}$.

In the remainder of this section, we show this nonapproximability result is tight to within a constant factor by presenting an approximation algorithm with a performance guarantee of $O(\log n)$. These results should be contrasted with the fact that under the symmetric power threshold model, there are constant factor approximation algorithms for the problem $[4,8]$.

The main idea behind the approximation algorithm is to reduce the problem to the computation of a connected dominating set for a graph with node weights. A simple observation allows us to restrict the class of solutions to the $\langle$ Undir, Connected, TotalP $\rangle$ problem. Consider a node $v_{i}$ and let $\gamma_{i}^{1} \leq \gamma_{i}^{2} \leq \ldots \leq \gamma_{i}^{n}$ denote the $n$ power threshold values in nondecreasing order from $v_{i}$ to the $n$ nodes of the system. We may assume without loss of generality that in any solution, the power value assigned to $v_{i}$ is one of $\gamma_{i}^{1}, \gamma_{i}^{2}, \ldots, \gamma_{i}^{n}$. This is because of the following:

(a) The power assigned to $v_{i}$ cannot be less than $\gamma_{i}^{1}$, since in such a case, $v_{i}$ cannot be adjacent to any other node in the induced graph.

(b) A power value which is greater than $\gamma_{i}^{j}$ but less than $\gamma_{i}^{j+1}$ for some $j$ can be replaced by $\gamma_{i}^{j}$ without deleting any edges in the induced graph.

(c) Similarly, a power value greater than $\gamma_{i}^{n}$ can be replaced by $\gamma_{i}^{n}$. 
1. Let $\alpha=6 \ln n \sum_{i=1}^{n} \gamma_{i}^{n}$.

2. From the given instance of $\langle$ UNDIR, CONNECTED, TOTALP $\rangle$, construct a graph $G_{1}\left(V_{1}, E_{1}\right)$ as follows.

(a) For each transceiver $v_{i}(1 \leq i \leq n)$ in the problem instance, create a set $g_{i}=\left\{u_{i}^{0}, u_{i}^{1}, \ldots, u_{i}^{n}\right\}$ of $n+1$ nodes. Let the weight $w\left(u_{i}^{0}\right)=\alpha$. For $1 \leq j \leq n$, let the weight $w\left(u_{i}^{j}\right)=\gamma_{i}^{j}$. The node set $V_{1}$ is given by $g_{1} \cup g_{2} \cup \ldots \cup g_{n}$.

(b) For each $i$, connect the nodes in $g_{i}$ together as an $(n+1)$-clique. (Nodes $u_{i}^{0}$, $1 \leq i \leq n$, are not involved in any edges other than these clique edges.)

(c) For any pair of nodes $u_{i}^{j}$ and $u_{k}^{l}$, where $1 \leq j, l \leq n$, if $\gamma_{i}^{j} \geq p\left(v_{i}, v_{k}\right)$ and $\gamma_{k}^{l} \geq p\left(v_{k}, v_{i}\right)$, then add the edge $\left\{u_{i}^{j}, u_{k}^{l}\right\}$ to $E_{1}$. The edge set $E_{1}$ consists of the clique edges added in Step 2(b) and the edges added in Step 2(c).

3. Use the algorithm of [7] using a small value (say, 0.1) for $\epsilon$ to find a connected dominating set $D_{1}$ of approximately minimal weight for $G_{1}$.

4. If for some $i, D_{1}$ contains both $u_{i}^{j}$ and $u_{i}^{k}$, where $j<k$, then delete $u_{i}^{j}$ from $D_{1}$. Let $D$ denote the resulting set after all such deletions have been carried out.

5. For each $i, 1 \leq i \leq n$, if $D$ contains $u_{i}^{j}$, then assign the power value $\gamma_{i}^{j}$ to $v_{i}$.

Fig. 2. Approximation Algorithm for 〈Undir, Connected, TotalP $\rangle$

A solution in which for every node $v_{i}$, the power value assigned is one of $\gamma_{i}^{1}$, $\gamma_{i}^{2}, \ldots, \gamma_{i}^{n}$ will be referred to as a canonical solution. Thus, we consider only canonical solutions in the remainder of this section.

Our approximation algorithm for the $\langle$ Undir, ConneCted, TotalP $\rangle$ problem under the asymmetric threshold model is shown in Figure 2. The algorithm constructs a graph $G_{1}\left(V_{1}, E_{1}\right)$ from the given instance of $\langle$ UNDIR, CoNNECTED, TOTALP $>$ and then invokes a known approximation algorithm for the minimum weighted connected dominating set problem [7]. We will prove the correctness and the performance guarantee of the algorithm through a series of lemmas.

For the remainder of this section, let $I$ denote the given instance of the $\langle$ Undir, Connected, TotalP $\rangle$ problem under the asymmetric threshold model, $f^{*}$ denotes an optimal power assignment to the nodes for this instance and $O P T(I)$ denotes the total power assigned by the chosen optimal solution. As before, $G_{f^{*}}$ denotes the graph induced by the optimal power assignment $f^{*}$.

For each transceiver $v_{i}$, the maximum power that can be assigned in a canonical solution is $\gamma_{i}^{n}, 1 \leq i \leq n$. Thus, we have the following observation.

Observation 1 OPT $(I) \leq \sum_{i=1}^{n} \gamma_{i}^{n}$.

Our next two lemmas relate $O P T(I)$, the weight of an optimal connected dominating set for $G_{1}$ and the weight of a connected dominating set produced by the approximation algorithm. For space reasons, the proofs of the lemmas are omitted.

Lemma 4. For the graph $G_{1}$ constructed in Step 2 of the algorithm, the weight of a minimum connected dominating set is at most OPT(I). 
Lemma 5. Consider the dominating set $D_{1}$ found in Step 3 of the algorithm (Figure 2).

(a) Let $W\left(D_{1}\right)$ denote the total weight of the nodes in $D_{1}$. Then, $W\left(D_{1}\right)<$ $6 \ln n O P T(I)$.

(b) $D_{1}$ does not contain any of the nodes $u_{1}^{0}, u_{2}^{0}, \ldots, u_{n}^{0}$.

(c) For every $i, 1 \leq i \leq n, D_{1}$ contains at least one of the nodes from the set $g_{i}-\left\{u_{i}^{0}\right\}$.

Lemma 6. The set of nodes $D$ computed in Step 4 of the algorithm is a connected dominating set of $G_{1}$. Further, for each $i, 1 \leq i \leq n, D$ contains exactly one vertex from the set $g_{i}-\left\{u_{i}^{0}\right\}$.

Proof: From Part (c) of Lemma 5, the dominating set $D_{1}$ contains at least one node from each group $g_{i}, 1 \leq i \leq n$. This property also holds for the set $D$ since Step 5 eliminates a node $u_{i}^{j}$ only when there is another node $u_{i}^{k}$ from the same group $g_{i}$. In other words, $D$ is also a dominating set for $G_{1}$.

Note also that for any two nodes $u_{i}^{j}$ and $u_{i}^{k}$ from the same group $g_{i}$, with $j<k$, the set of nodes to which $u_{i}^{j}$ is adjacent is a subset of the corresponding set for $u_{i}^{k}$. Thus, the set of nodes remains a connected dominating set even after $u_{i}^{j}$ is deleted. In other words, $D$ is a connected dominating set for $G_{1}$.

It is easy to see that the approximation algorithm of Figure 2 runs in polynomial time. We now establish its correctness and performance guarantee.

Theorem 4. The power assignment produced by the algorithm induces a connected graph. Further, the algorithm provides a performance guarantee of $O(\log n)$.

Proof: From Lemma 6 , the set $D$ contains exactly one node from $g_{i}-\left\{u_{i}^{0}\right\}$, for each group $g_{i}$. Therefore, Step 5 of the algorithm assigns a power value to each transceiver. Since $D$ is a connected dominating set and there is a one-to-one correspondence between $D$ and the set of transceivers, the graph induced by the power assignment is also connected.

The total power assigned to all the nodes is equal to $W(D)$, the total weight of all the nodes in $D$. By Part (a) of Lemma $5, W\left(D_{1}\right)<6 \ln n O P T(I)$. Since $D \subseteq$ $D_{1}$ and the node weights are nonnegative, it follows that $W(D)<6 \ln n O P T(I)$. In other words, the approximation algorithm has a performance guarantee of $O(\log n)$.

\section{Open Problems}

Our work raises several open questions. First, it would be of interest to investigate whether there is a bicriteria approximation algorithm for the 〈UNDIR, DIAMETER, TOTALP $\rangle$ problem with a performance guarantee of $(O(1), O(\log n))$ for any given diameter value. A second problem is to improve the approximation ratio for the $\langle$ Undir, Deg LB, TotalP $\rangle$ problem. Finally, it would also be of interest to consider other topology control problems under the asymmetric threshold model. 


\section{References}

1. D. M. Blough, M. Leoncini, G. Resta, and P. Santi, "On the Symmetric Range Assignment Problem in Wireless Ad Hoc Networks", Proc. 2nd IFIP International Conference on Theoretical Computer Science, Montreal, August 2002.

2. M. Charikar, C. Chekuri, T. Cheung, Z. Dai, A. Goel, S. Guha, and M. Li, "Approximation Algorithms for Directed Steiner Problems", Journal of Algorithms, Vol. 33, No. 1, 1999, pp. 73-91.

3. W. Chen and N. Huang, "The Strongly Connecting Problem on Multihop Packet Radio Networks", IEEE Trans. Communication, Vol. 37, No. 3, Mar. 1989.

4. G. Calinescu, I. Mandoiu, and A. Zelikovsky. "Symmetric Connectivity with Minimum Power Consumption in Radio Networks", Proc. 2nd IFIP International Conference on Theoretical Computer Science, Montreal, August 2002.

5. A. E. F. Clementi, P. Penna and R. Silvestri. "Hardness Results for the Power Range Assignment Problem in Packet Radio Networks", Proc. Third International Workshop on Randomization and Approximation in Computer Science (APPROX 1999), Lecture Notes in Computer Science Vol. 1671, Springer-Verlag, July 1999, pp. 195-208.

6. G. Calinescu and P-J Wan. "Symmetric High Connectivity with Minimum Total Power Consumption in Multihop Packet Radio Networks", Submitted for journal publication, 2003.

7. S. Guha and S. Khuller, "Improved Methods for Approximating Node Weighted Steiner Trees and Connected Dominating Sets", Information and Computation, Vol. 150, 1999, pp. 57-74.

8. L. M. Kirousis, E. Kranakis, D. Krizanc and A. Pelc, "Power Consumption in Packet Radio Networks", Proc. 14th Annual Symposium on Theoretical Aspects of Computer Science (STACS 97), Lecture Notes in Computer Science Vol. 1200, Springer-Verlag, Feb. 1997, pp. 363-374.

9. G. Kortsarz and D. Peleg, "Approximating the Weight of Shallow Light Trees", Discrete Applied Mathematics, Vol. 93, 1999, pp. 265-285. (Preliminary version appeared in Proc. Eighth ACM-SIAM Symp. on Discrete Algorithms (SODA'97), New Orleans, LA, Jan. 1977, pp. 103-110.)

10. E. L. Lloyd, R. Liu, M. V. Marathe, R. Ramanathan and S. S. Ravi, "Algorithmic Aspects of Topology Control Problems for Ad hoc Networks", Proc. Third ACM International Symposium on Mobile Ad Hoc Networking and Computing (MobiHoc'02), Laussane, Switzerland, June 2002.

11. M. V. Marathe, R. Ravi, R. Sundaram, S. S. Ravi, D. J. Rosenkrantz and H. B. Hunt III, "Bicriteria Network Design Problems", Journal of Algorithms, Vol. 28, No. 1, July 1998, pp. 142-171.

12. R. Ramanathan, "On the Performance of Ad Hoc Networks with Beamforming Antennas", Proc. Second ACM International Symposium on Mobile Ad Hoc Networking and Computing (MobiHoc'01), Long Beach, CA, Oct. 2001.

13. R. Ramanathan and R. Rosales-Hain, "Topology Control of Multihop Wireless Networks Using Transmit Power Adjustment", Proc. INFOCOM 2000. 